\title{
Diffractive optic fluid shear stress sensor
}

\author{
Darius Modarress, Dominique Fourguette and Frederic Taugwalder \\ VioSense Corporation, 36 S. Chester Avenue, Pasadena, CA 91106 \\ Tel: (626)432-9950, Fax: : (626)432-1996,E-mail: modarress@viosense.com \\ Manooch Koochesfahani \\ Michigan State University(address?) \\ Tel: (517) 353-5311, Fax??,E-mail: koochesf@egr.msu.edu \\ Morteza Gharib \\ Graduate Aeronautics Laboratory, California Institute of Technology,1200 E. California Blvd., Pasadena, CA 91125 \\ Tel: (818) 393-3548, Fax: (818) 393-4540,E-mail: mory@caltech.edu \\ Daniel W. Wilson \\ Jet Propulsion Laboratory, California Institute of Technology, MS 302-231, 4800 Oak Grove Drive, Pasadena, CA 91109 \\ Tel: (818) 393-3548, Fax: (818) 393-4540, E-mail: daniel.w.wilson@jpl.nasa.gov
}

\begin{abstract}
Recent advances in the design and fabrication of integrated diffractive optical elements has resulted in the development of a small and integrated micro-optical shear stress sensor. Miniaturization of these sensors has been achieved by integrating the collective function of a large number of refractive optical elements onto a single substrate without the loss of functionality and efficiency. This paper describes the fabrication of the micro-shear stress sensor and an experiment designed for the evaluation of its performance. The results show that, the micro-optical shear stress sensor accurately measures the wall velocity gradient for all laminar flows and for turbulent flows with moderate Reynolds numbers.
\end{abstract}

\section{Introduction}

Accurate measurement of the wall shear stress is needed for a number of aerodynamic studies. Shear forces generated by liquids or gases flowing over solid surfaces can significantly influence the performance of aircraft, ships, or surface-transport vehicles. Techniques that have been used for the measurement of shear stress include surface mounted force balance, surface mounted hot wire, surface mounted hot plate, MEMS hot-film, and oil film interferometery. The surface mounted force balance directly measures the shear force on the body. This technique requires a floating element, is usually not very small and has a limited frequency response. The hot wire, hot plate and MEMS hotfilm techniques all require calibration and are not generally suitable for liquid flows. The oil film technique has been used for aerodynamic applications, but has a limited utility in liquid flows.

The optical shear stress sensor reported here was developed based on a technique first presented by Naqwi and Reynolds [1] using conventional optics. The concept was based on the use of a modified laser Doppler anemometry (LDA) with a set of diverging fringes located close to the wall and within the linear region of the boundary layer. The developed sensor provided an integrated diffractive optical element (doe) sensor that integrated the transmitter and receiver optics on the same substrate, as shown in Figure 1. The surface mounted sensor generates a pattern of diverging interference fringes, originated at the surface, and extended into the boundary layer region. As in the LDA technique, the scattered light from the particles passing through the fringes is collected through a window at the surface of the sensor. The intersection region of the transmission fringe pattern and the receiver field of view defined by the backscatter collection optics defined the overall measurement volume location and dimension. 


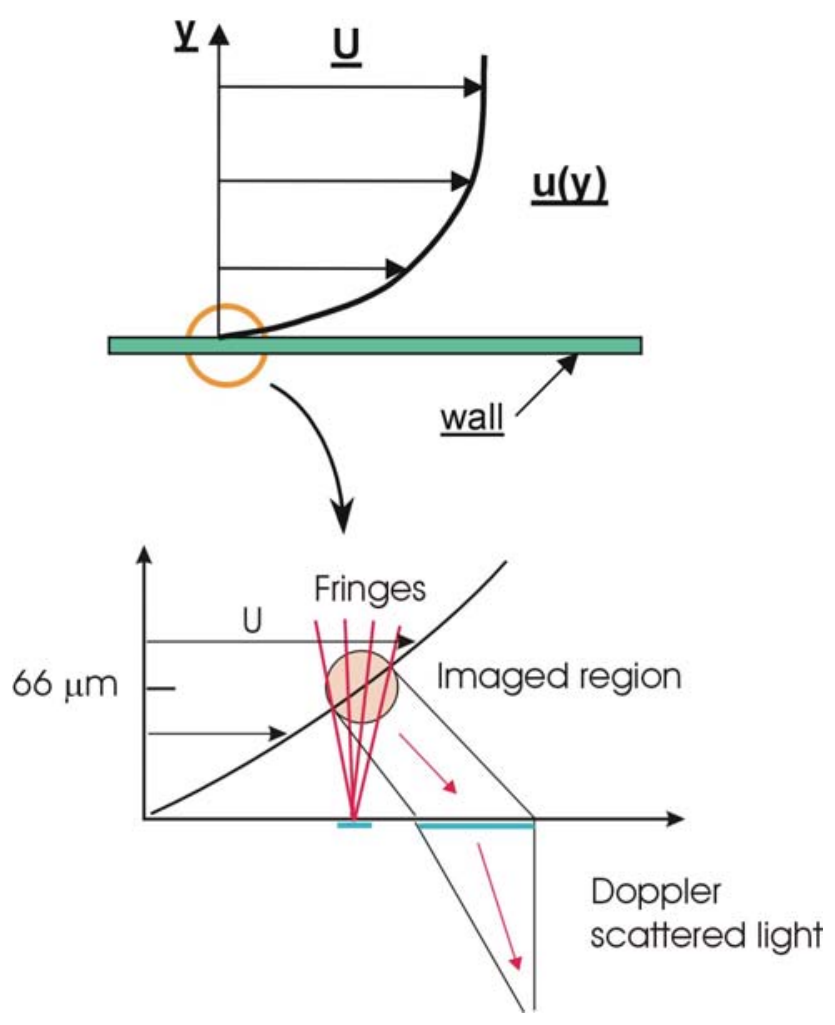

Figure 1. Schematic of the Micro-shear stress sensor

The local fringe separation, $\delta$, was designed to be linear with the distance from the sensor, y, given by $\delta=k \times y$, where $k$ is the fringe divergence rate. As particles in the fluid flow through the linearly diverging fringes, they scatter light with a frequency $f$ that is proportional to the instantaneous velocity, u, and inversely proportional to the fringe separation at the location of particle trajectory.

$f=\frac{u}{\delta}=(1 / k) \frac{u}{y}$.

In the linear sub-layer region of the boundary layer where,

$\left.\frac{\partial u}{\partial y}\right|_{w} \equiv \frac{u}{y}$,

the measured Doppler frequency, $\mathrm{f}$, simply multiplied by the fringe divergence yields the velocity gradient at the wall.

$f \times k=\left.\frac{\partial u}{\partial y}\right|_{w}$,

which is proportional to the wall shear.

\section{Design and modeling}

A conceptual drawing of the micro-shear stress sensor is shown in Figure 2. The diverging light from a diode laser is focused by a diffractive optical element (DOE) to two parallel line foci. These foci are coincident with two slits in a metal mask on the opposite side of a quartz substrate. The light diffracts from the slits and interferes to form linearly diverging fringes to a good approximation. The light scattered by particles traveling through the fringe pattern is collected through a window in the metal mask. Another DOE on the backside focuses the light to an optical fiber connected to a detector. 


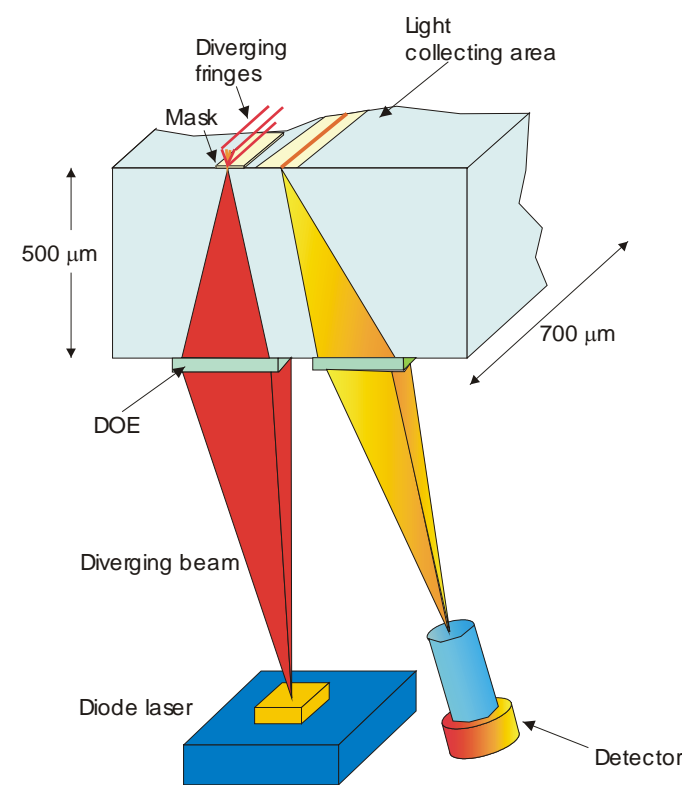

Figure 2. Schematic of the shear stress sensor assembly.

A series of simulations were performed to aid in the design of the sensor. A finite-difference simulation of the fringe pattern for $2 \mu \mathrm{m}$ wide slits separated by $10 \mu \mathrm{m}$ is shown in Figure 3. The fringe pattern displays a suitable number of fringes for adequate measurements. The number of high-contrast fringes is determined by the slit width and the divergence of the fringe pattern is determined by the slit separation.

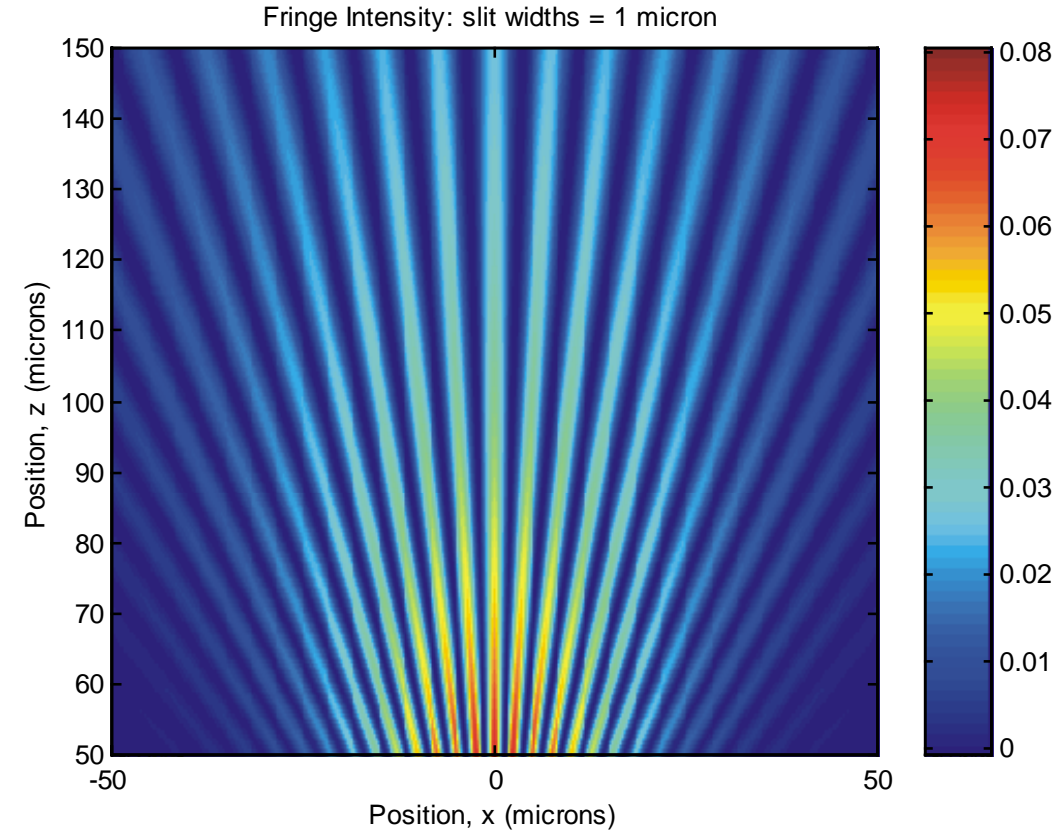




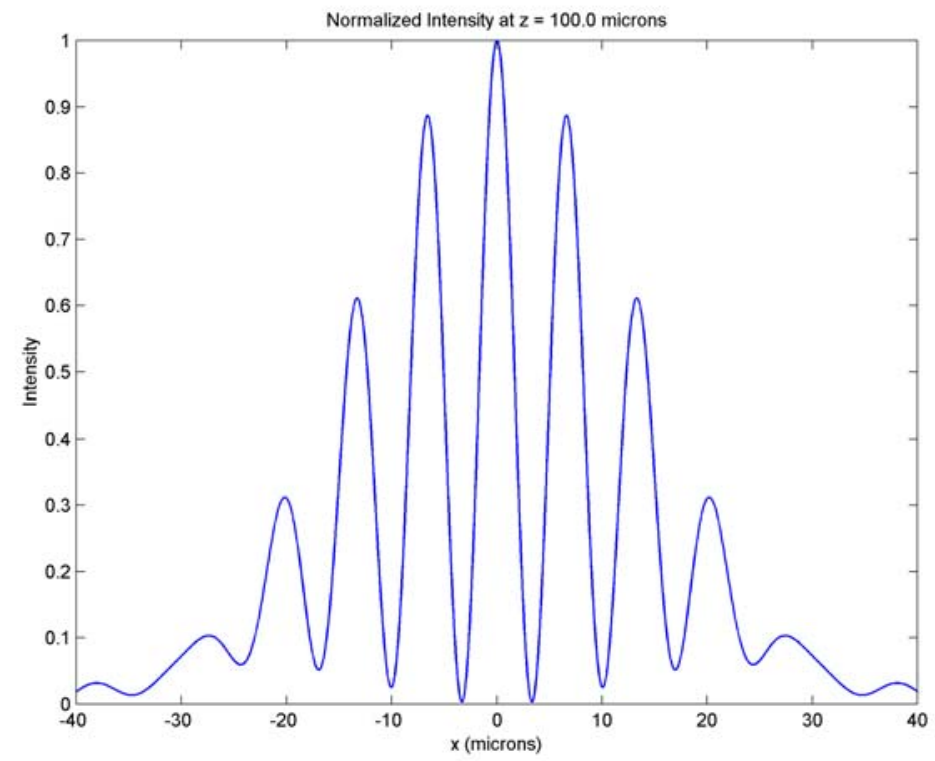

Figure 3. Fringe pattern resulting from $2 \mu \mathrm{m}$ slits separated by $10 \mu \mathrm{m}$ (propagation of a finite-difference solution of slit diffraction when illumined by the dual-line-focus laser lens).

\section{Fabrication and testing}

The main sensor element was fabricated by two-sided lithography on a $500 \mu \mathrm{m}$ thick quartz substrate. The slits and collecting window on the front were fabricated by direct-write electron-beam lithography followed by wet etching of evaporated chrome. The polymethyl methacrylate (PMMA) diffractive optical elements on the back were fabricated by analog direct-write electron-beam lithography followed by acetone development [2]. A photograph and atomic force microscope scan of the dual-line focus-laser lens are shown in Fig. 4.
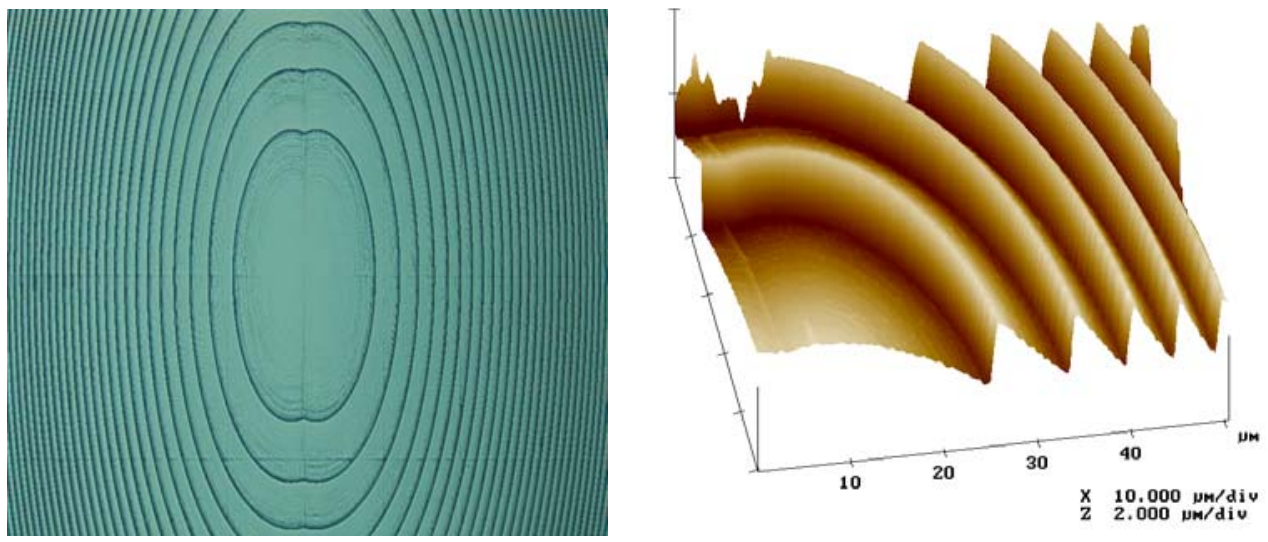

Figure 4. Photograph (left) and AFM scan (right) of the center of the dual-line-focus laser lens 
The shear stress sensor's elements were assembled into a package (Fig. 5) with a diode laser (660 nm) and a port for the collection fiber. The overall size of this prototype is $15 \mathrm{~mm}$ in diameter and $20 \mathrm{~mm}$ in length. The fringes were imaged with a CCD camera using a microscope objective and are shown in Fig. 5. The fringe divergence was measured to be linear with a slope in close agreement with theory. The contrast is very satisfactory and preliminary tests using a moving surface through the fringe pattern yield a clear signal. Testing of the receiver side of the sensor element is underway.
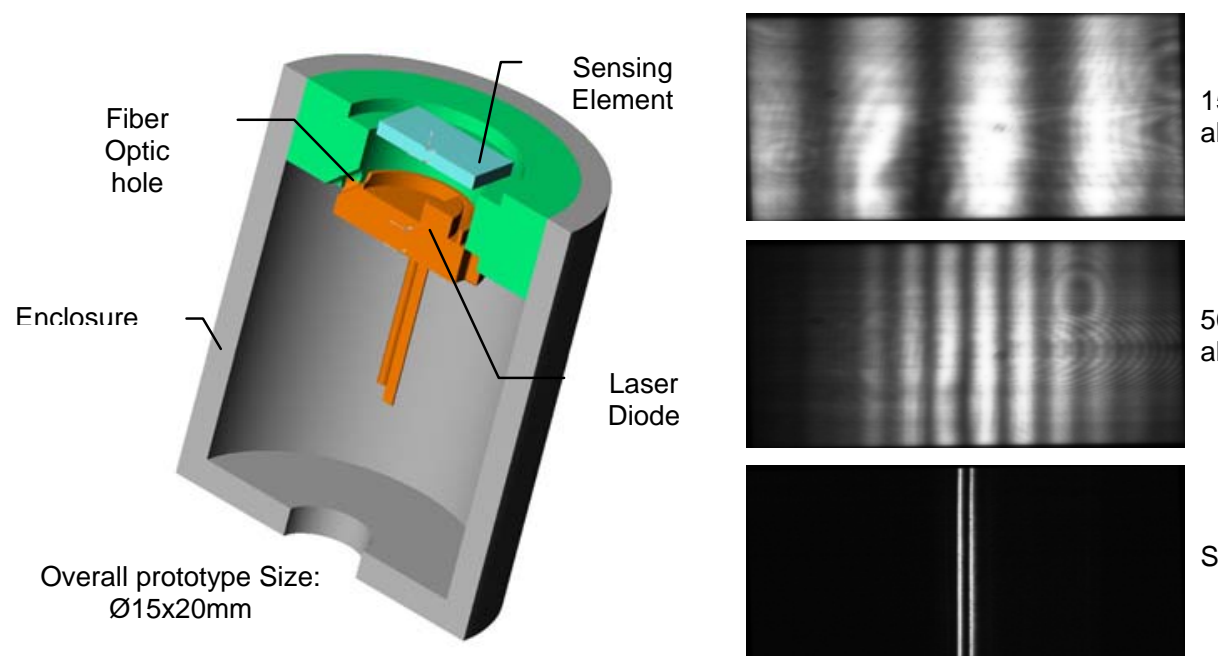

150 microns above surface

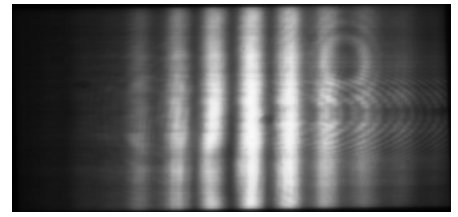

50 microns above surface

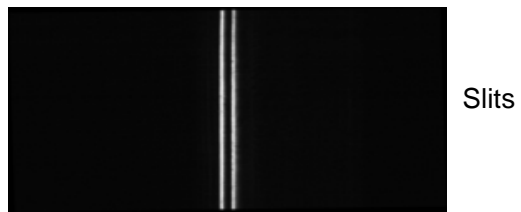

Figure 5. Shear stress sensor assembly (left) and photographs of the fringes at different heights above the surface (right).

A photograph of the shear stress sensor is shown in Figure 6. The sensor substrate, shown in, is mounted into the sensor element location shown on the front face of the assembly. The diverging fringe pattern is visible away from the surface. In this configuration, the light source and the detection electronics are located remotely and are connected to the sensor through optical fibers.

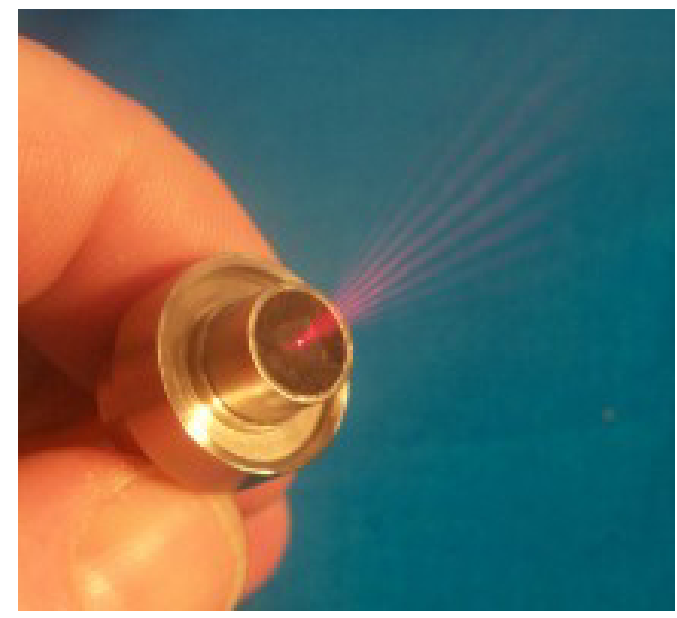

Figure 6. Micro-optical shear stress sensor

\section{Experimental Results.}

A miniature LDA and two shear stress sensors were installed on a flat plate in a small water tunnel facility at the Graduate Aeronautics Laboratory of the California Institute of Technology. The facility is equipped with a $90 \mathrm{~cm}$ long test section. The traversing miniature LDA and a shear stress sensor were mounted on a flat plate at $\mathrm{x}=34.93 \mathrm{~cm}$ and $39.37 \mathrm{~cm}$ from the leading edge, as shown in Figure 


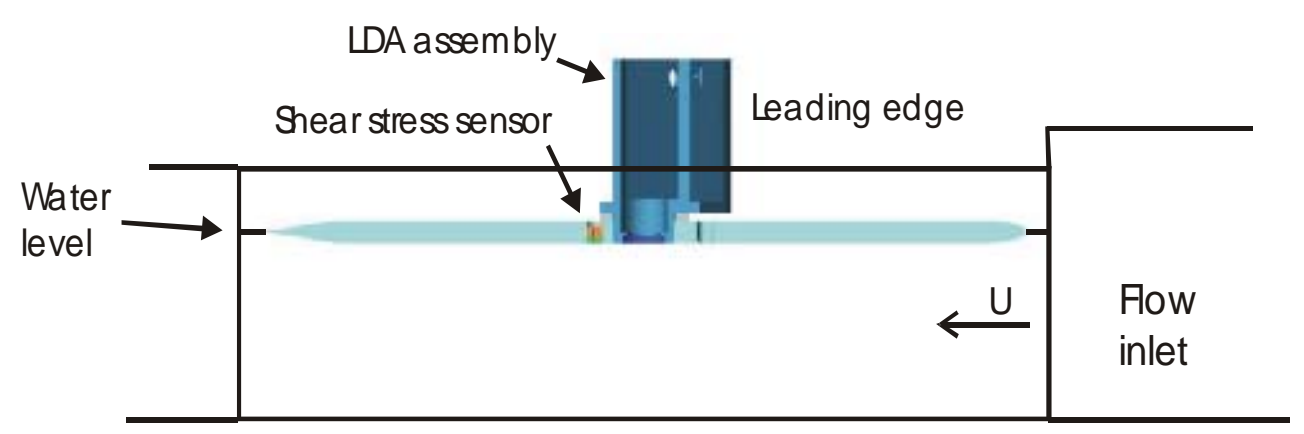

Figure 7. Schematic of the test section with the flat plate.

Measurements were made at free-stream velocities of $\mathrm{U}_{0}=18.1$ and $\mathrm{U}_{0}=26.9 \mathrm{~cm} / \mathrm{s}$. Velocity surveys were conducted with the Miniature LDA and the data are shown in Figure 8. A Blasius profile was fitted to the data. Closer examination of the data indicated that the measured velocity at the point closest to the wall was slightly larger than the corresponding value for the Blasius profile. The same conclusion was arrived at for both run cases. This variation was of important consequence when the results of the micro shear stress was compared with the wall shear estimated from the velocity profile.

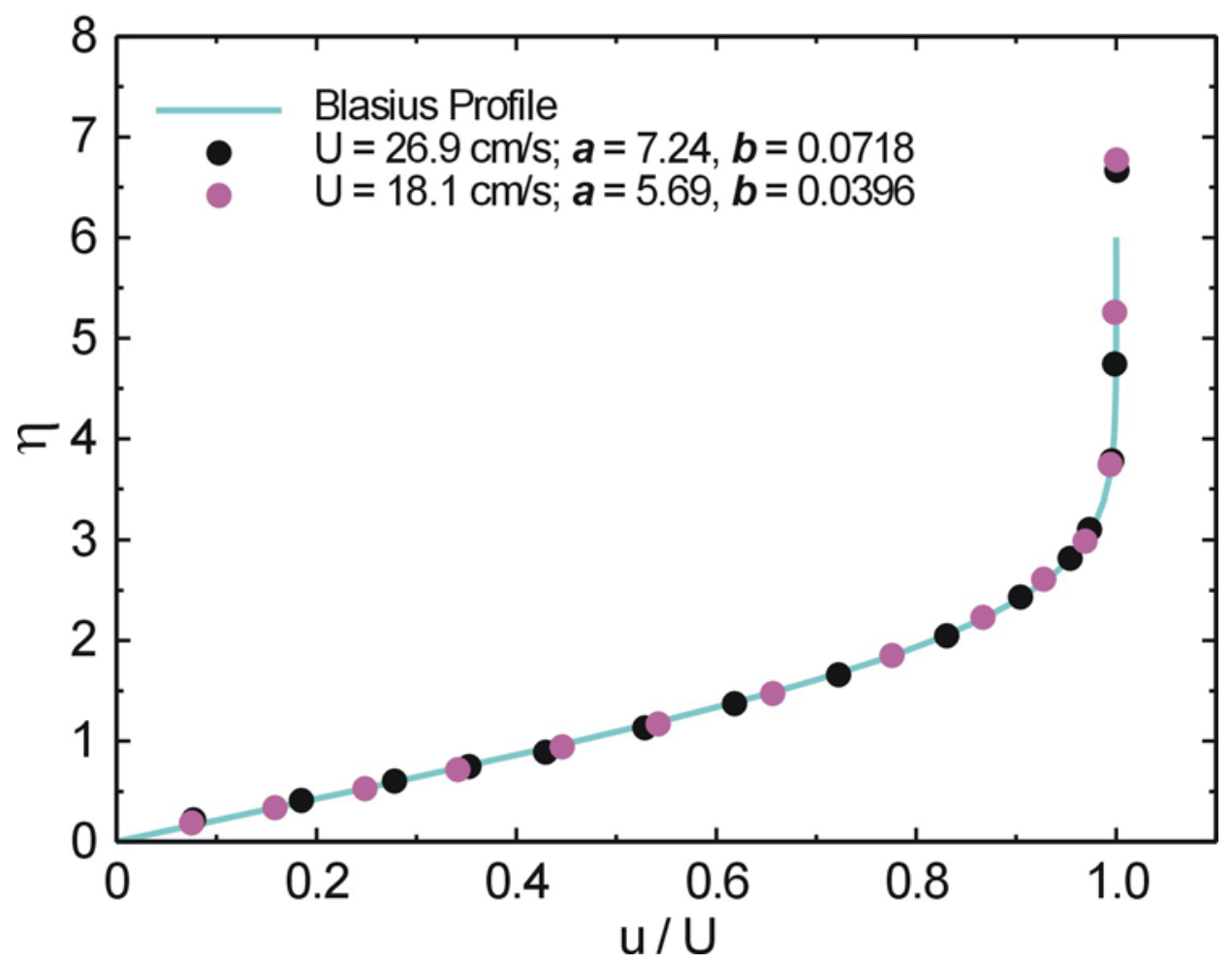

Figure 8. Results of the velocity survey using the miniature LDA for $\mathrm{U}_{0}=18.1 \mathrm{~cm} / \mathrm{s}$.

The data obtained from the micro- shear stress was compared with the wall shear estimated from the velocity profiles. The wall velocity gradient was estimated using two methods. The first method involved calculating the velocity gradient at the wall using the last two data points of the velocity 
survey and the second method involved obtaining the velocity gradient at the wall using the curve fit. Figure 9 shows the comparison of wall velocity gradients. The gradient obtained from the slope of the velocity data agrees well with the micro-shear stress data. However both gradients were greater than that calculated from the Blasius fit. This result led us to postulate the presence of a slightly favorable pressure gradient at the measurement location. A Falkner-Skan fit of the boundary layer profile shown in Figure 10 reveals a weak favorable pressure gradient.

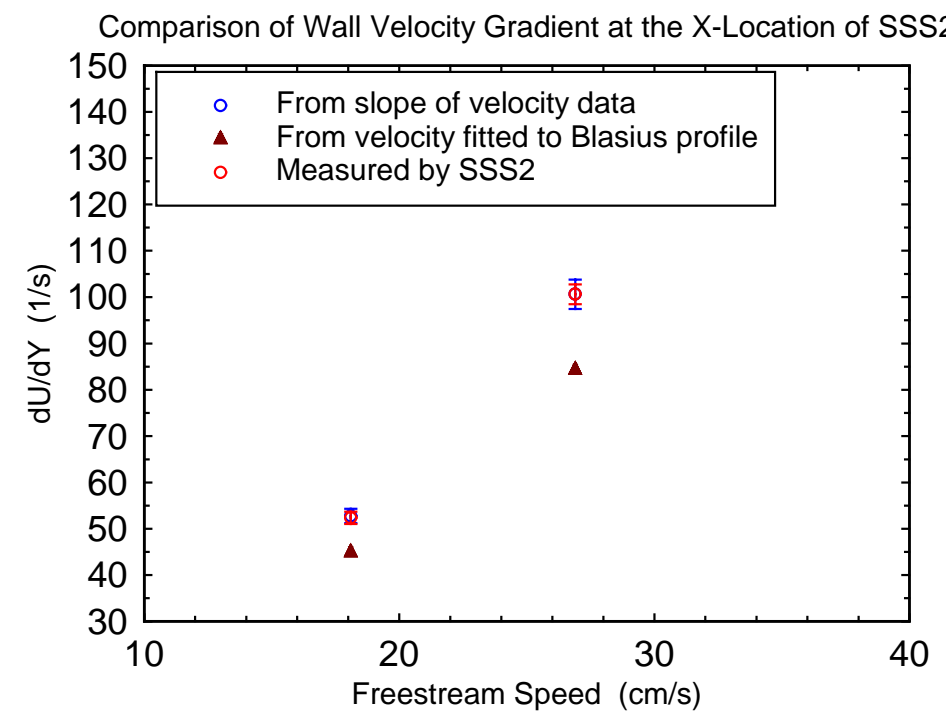

Figure 9. Comparison of wall velocity gradients obtained with the miniature LDA and the microshear stress sensor.

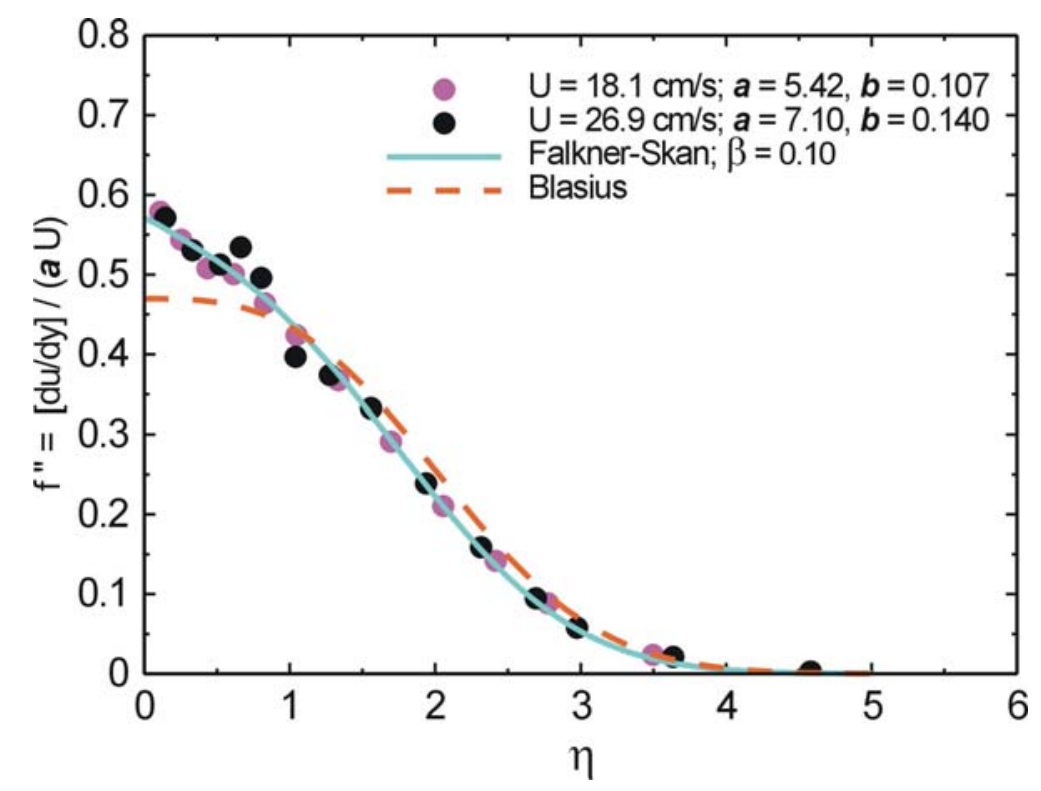

Figure 10 Falkner-Skan fit to the boundary layer velocity profile 


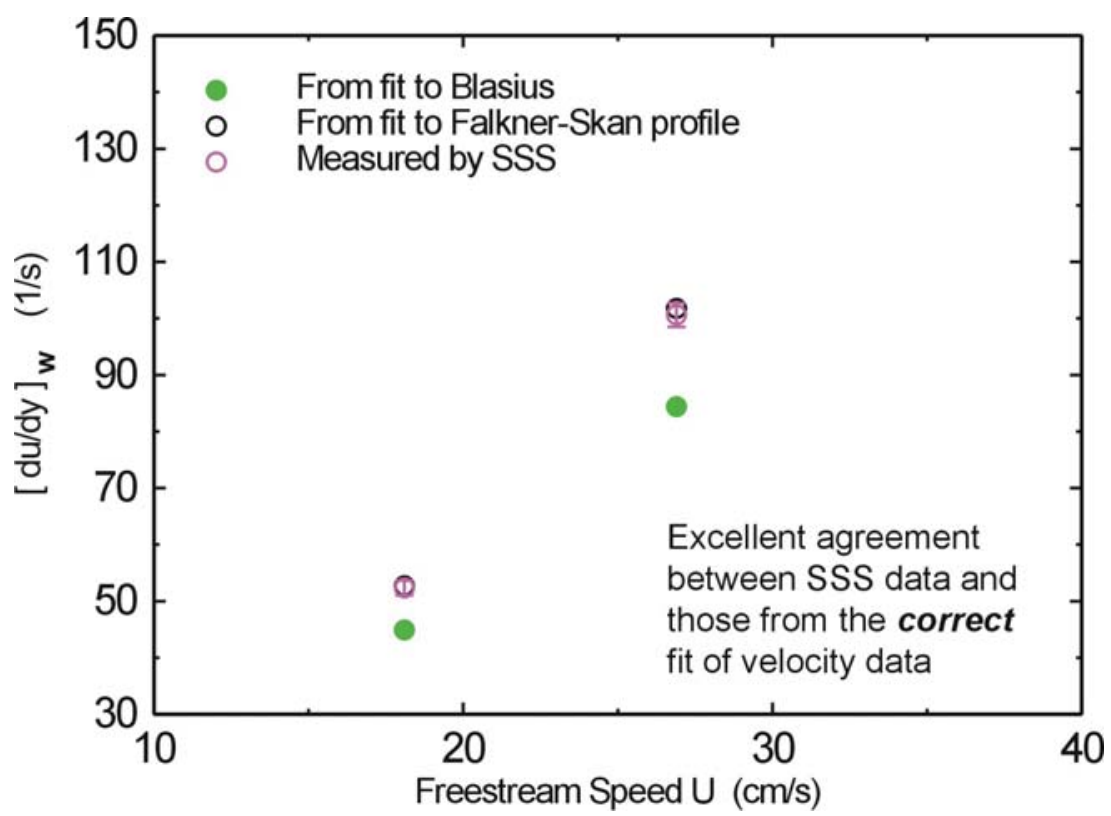

Figure 11 Velocity gradient at the wall obtained from a Blasius, fit, and Falkner-Skan fit and direct measurement with the wall shear stress sensor

Ideally, accurate measurement of the wall shear stress with the micro-shear stress sensor requires that the probe volume to be within the linear region of the boundary layer. An estimate of the measurement errors as a function of the flow Reynolds number was calculated for the flow on a flat plate in the absence of a pressure gradient for this particular shear stress sensor. Figure 12 shows the error bounds for a laminar boundary layer and Figure 13 shows the results for a turbulent boundary layer.

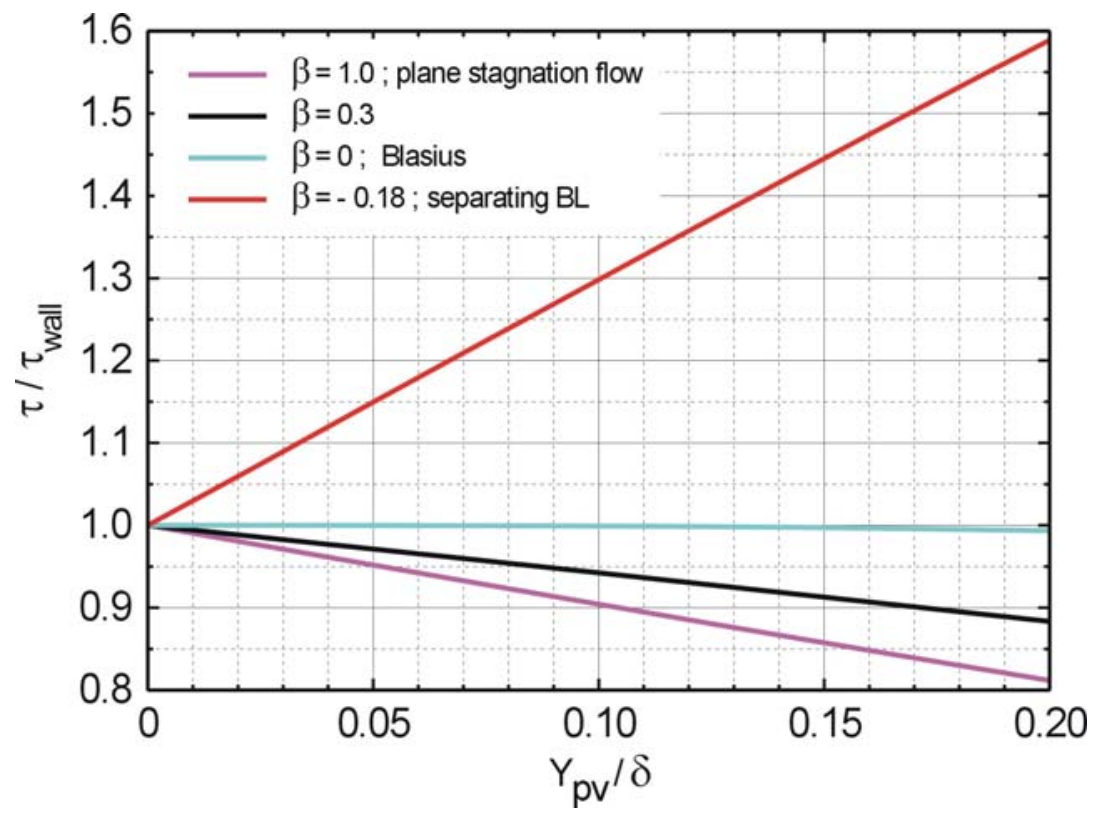

Figure 12. Limit of accuracy of the shear stress sensor in laminar boundary layer

In both plots, $\mathrm{Y}_{\mathrm{pv}}$ represents the location of the probe volume above the surface. The accuracy of 
the measurement is better than $99 \%$ for experimental conditions above the blue line. For instance, the sensor described in this paper $\left(\mathrm{Y}_{\mathrm{pv}}=60\right.$ microns) will yield accurate results for a Reynolds number $=10^{6}$ when located $1 \mathrm{~m}$ downstream from the leading edge of the plate. The error plots show that for turbulent flows, the measurement errors increase rapidly with the Reynolds number. Therefore, it is important that the sensor to be fabricated such that the probe volumes remain within the laminar sub-layer at all times. New shear stress sensor designs are currently under development to extend the operating envelope of these sensors.

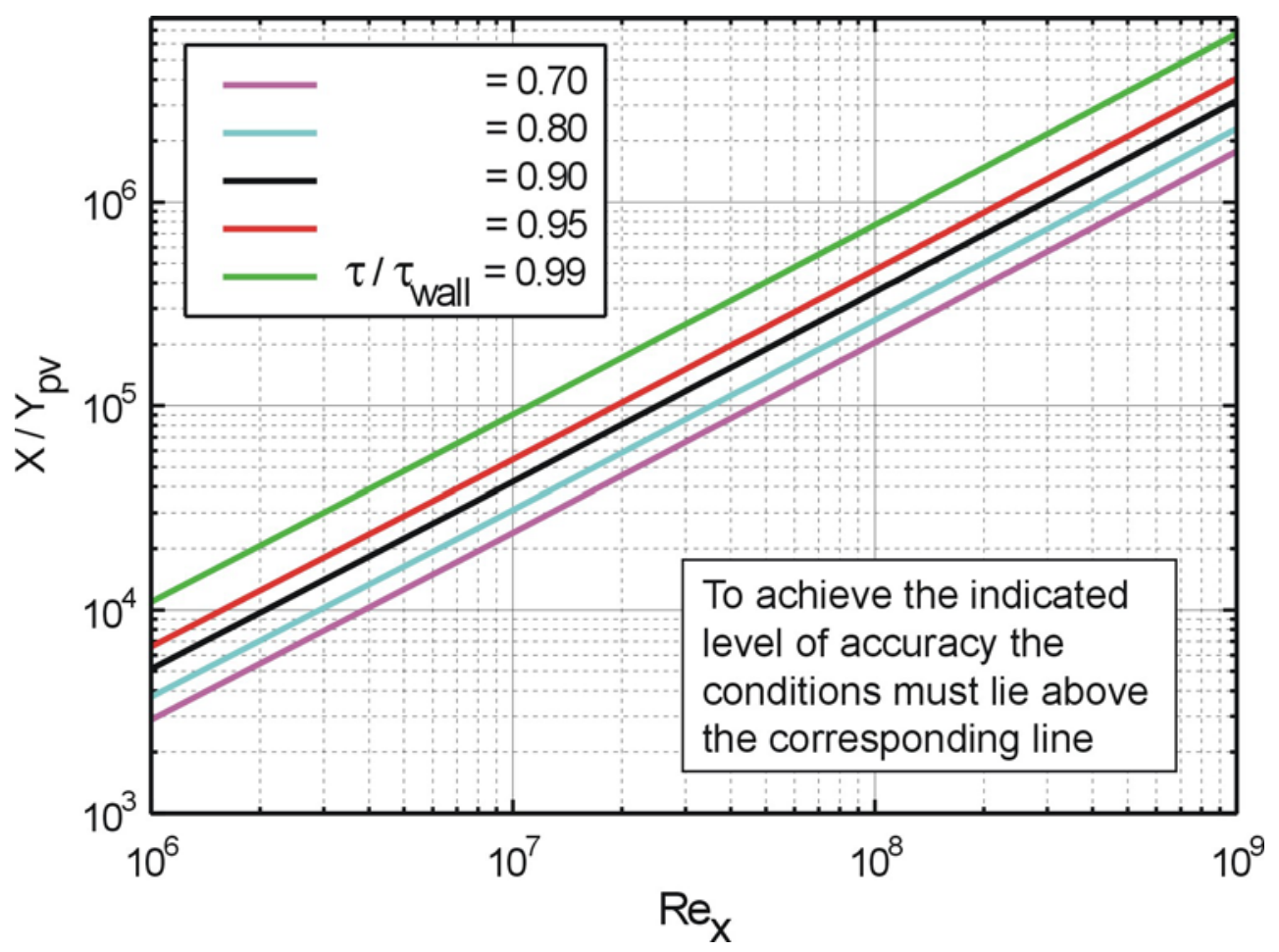

Figure 13. Limit of accuracy of the shear stress sensor in turbulent boundary layers.

A second generation Micro-shear stress sensor has been designed for the applications in high Reynolds number flows as shown in Figure 14. It uses multiple sensors located at multiple locations relative to the wall. It is based on the assumption that the fringes no longer require to be located in the linear sublayer of the boundary layer. By having two simultaneous measurement of the flow velocities, the near wall boundary layer model could be used to provide a more accurate estimates of the wall shear stress. 


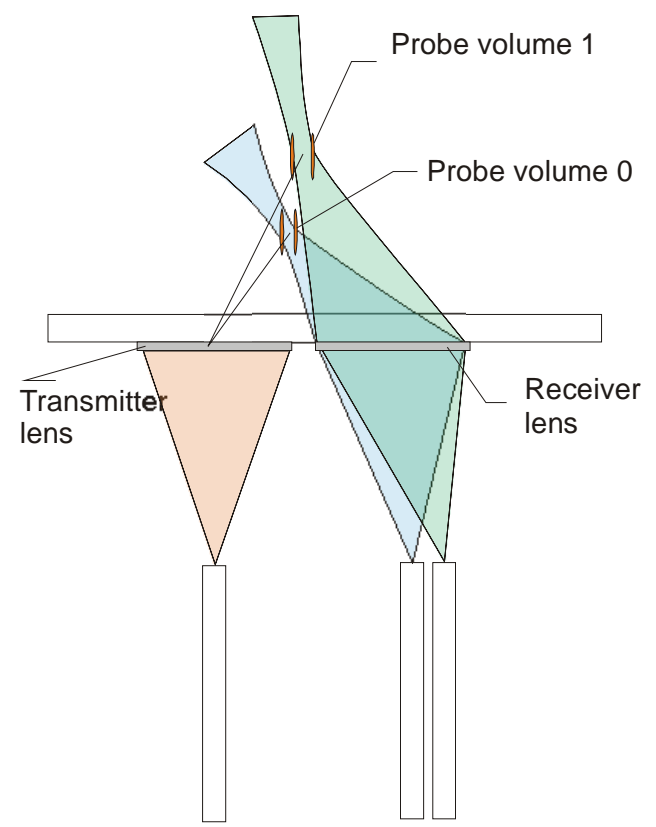

Figure 14. Conceptual design of a wall shear stress sensor for high Reynolds numbers

\section{Conclusions}

Recent progress in the miniaturization technology has allowed the development of a small optical wall mounted shear stress sensor. Significant steps in the concept development and fabrication of the shear stress sensor stemming from most recent developments in micro device technology were achieved and results obtained in a test facility were reported. The data obtained in a laminar boundary layer with a weak favorable pressure gradient. Excellent agreement between the wall velocity gradient calculated from the boundary layer velocity survey performed with the Mini LDA and the measurements obtained with the micro-shear stress sensor was achieved. The errors associated with the boundary layer thickness for laminar flows and the flow Reynolds number for turbulent flows are presented. The results will serve as an indicator for the applicability of the microshear stress sensor to different flow regimes.

\section{Acknowledgements}

The reported research was supported by ONR, under the Grant number N66001-99-1-8902 and DARPA and SPAWAR, under the Grant number N66001-99-1-8902 to Caltech.

\section{$\underline{\text { References }}$}

1. Naqwi, A. A., Reynolds, W.C., (1987) "Dual cylindrical wave laser-Doppler method for measurement of skin friction in fluid flow," Report No. TF-28, Stanford University.

2. P. D. Maker, D. W. Wilson, and R. E. Muller, "Fabrication and performance of optical interconnect analog phase holograms made be E-beam lithography," in Optoelectronic Interconnects and Packaging, R. T. Chen and P. S. Guilfoyle, eds., Proc. SPIE CR62, 415430 (1996). 